\title{
Pain management following robotic thoracic surgery
}

\section{Farid Gharagozloo}

Center for Advanced Thoracic Surgery, Global Robotics Institute, Advent Health Celebration, University of Central Florida, Celebration, FL 34786, USA.

Correspondence to: Dr. Farid Gharagozloo, Center for Advanced Thoracic Surgery, Global Robotics Institute, Advent Health Celebration, University of Central Florida, 400 Celebration Place, Celebration, FL 34786, USA. E-mail: gharagozloof@aol.com

How to cite this article: Gharagozloo F. Pain management following robotic thoracic surgery. Mini-invasive Surg 2020;4:8. http://dx.doi.org/10.20517/2574-1225.2019.62

Received: 19 Dec 2019 First Decision: 13 Jan 2020 Revised: 14 Jan 2020 Accepted: 17 Jan 2020 Published: 12 Feb 2020

Science Editor: Noriyoshi Sawabata Copy Editor: Jing-Wen Zhang Production Editor: Jing Yu

\begin{abstract}
For robotic thoracic surgical patients, minimizing pulmonary complications is the key to decreasing morbidity. Once the pain is controlled, the morbidity associated with thoracic surgery is decreased. Consequently, control of pain is the core requirement in robotic thoracic surgical patients. Appropriate pain control depends on a multifaceted program that is based on an understanding of the pathophysiology of pain. A multifaceted pain control program after robotic surgery needs to address local and systemic pain pathways. This review outlines such a multifaceted program with the use of subpleural catheters for prolonged ambulatory infusion of local anesthetic for 10 days, nonsteroidal anti-inflammatory agents, and measured use of narcotic analgesics.
\end{abstract}

Keywords: Robotic surgery, pain management, analgesia, subpleural catheters, on-Q

\section{INTRODUCTION}

Although it is hypothesized that robotic surgery is associated with lower pain-related morbidity, it is important to address pain in the patient undergoing robotic surgery as diligently as a patient undergoing any other thoracic surgical procedure. Unlike the abdomen, even the most minimally invasive procedures on the chest can be painful. In addition, the nature and severity of the thoracic pain experience for each individual patient is highly subjective and complex. Therefore, regardless of the number and type of incisions or ports, acute and chronic pain associated with robotic thoracic surgical procedures should be recognized and treated aggressively.

\footnotetext{
(@) (

(C) The Author(s) 2020. Open Access This article is licensed under a Creative Commons Attribution 4.0 International License (https://creativecommons.org/licenses/by/4.0/), which permits unrestricted use, sharing, adaptation, distribution and reproduction in any medium or format, for any purpose, even commercially, as long as you give appropriate credit to the original author(s) and the source, provide a link to the Creative Commons license, and indicate if changes were made.
}

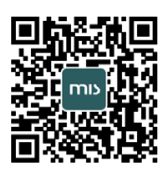


For robotic thoracic surgical patients, minimizing pulmonary complications is the key to decreasing morbidity. Studies have shown that simple deep breathing and coughing in the postoperative period can effectively prevent complications such as atelectasis and pneumonia. The ultimate goal is to clear secretions, maintain expansion of the lung, and decrease the complications associated with pulmonary collapse. This goal is achieved by the patient's ability to cough and deep breath, as well as the adjunctive measures of spirometry, chest physiotherapy, and bronchoscopy. In turn, effective clearance of secretions with cough and early mobilization are attained primarily by optimal pain control. Control of pain is the core requirement for all postoperative measures in robotic thoracic surgical patients. Once the pain is controlled, the morbidity associated with thoracic surgery is decreased.

\section{PATHOPHYSIOLOGY OF PAIN}

As defined by the International Association for the Study of Pain, pain is both the sensory and the emotional experience that is associated with actual or potential tissue damage, and it is described in terms of that damage ${ }^{[1]}$. The unique and individual nature of pain perception stems from the fact that the sensory experience is also associated with an individual's affective and cognitive response. As a result of the complex interaction between the pain stimulant and the individual's unique response to the stimulant, the cause and effect relationship between actual tissue damage and perception of pain is not constant among individuals.

The pain pathway begins with nociception. Nociception is the process whereby certain stimuli (chemical, mechanical, or thermal) activate a specific physiologic neural pathway. Nociceptors are the peripheral nerve endings of sensory neurons and supply skin, muscles, joints, and other tissues. These nerve endings are attached to axons, which communicate with the spinal cord or brainstem nuclei. The faster conducting myelinated axons or A-delta fibers are responsible for the shorter-lived but higher-intensity pain sometimes referred to as "first pain". The slower conducting unmyelinated axons or C fibers produce the duller and more prolonged pain sensation known as "second pain",[2].

Four processes lead to pain perception: (1) transduction; (2) transmission; (3) modulation; and (4) perception.

(1) Transduction: Transduction takes place in the peripheral nerve endings, where a stimulus is converted to electrical activity.

(2) Transmission: During transmission, the electrical activity is conducted through the nervous system. Axons from peripheral sensory neurons transmit impulses to the spinal cord, where they synapse with second-order neurons. Spinal second-order neurons project to different brainstem and diencephalic structures. In turn, neurons from these structures project to the various cortical sites responsible for sensation.

(3) Modulation: During modulation, the neural input, and thereby the pain process, is altered. Modulation occurs in the dorsal horn of the spinal cord.

(4) Perception: During the phase of perception, the neural activity in a somatosensory pathway results in the subjective sensation of pain. Perception results from the activation of primary and secondary somatosensory and limbic cortices ${ }^{[3]}$.

With tissue damage, nociceptors are stimulated. The initial stimulation of the nociceptors as the result of tissue damage leads to enhanced response of these receptors and increased sensitivity to further stimuli. 
Consequently, stimulated nociceptors are upregulated and become more responsive to further stimuli. Hyperalgesia refers to the phenomenon by which stimulated nociceptors become more sensitive to further stimuli. In addition to upregulation from the original stimulus, several humoral pathways enhance the effect of the painful stimulus. Tissue damage, such as with any incision, releases certain mediators, e.g., bradykinin, potassium, calcitonin gene-related peptide, and prostanoids such as prostaglandins and leukotrienes ${ }^{[1]}$. Substance $\mathrm{P}$ is also released. Substance $\mathrm{P}$ acts on mast cells to induce degranulation with the resultant release of histamine. All these activate and sensitize nociceptors. Substance P also dilates blood vessels, causing edema, and releasing more bradykinin ${ }^{[4]}$. Combination of the humoral mediators results in a decrease in the activation threshold and enhances the sensitivity of the nociceptors to further stimuli. In addition, the "cascade" effect results in increased nerve sensitivity over a much wider field than the original injury.

Understanding the peripheral pathways and the chemical mediators is important in devising techniques for pain control. For example, peripheral opioid receptors are uncovered in response to inflammation. These receptors are the target for endogenous opioids, which are released locally by the immune system. Binding these opioid receptors acts to decrease nociceptor output ${ }^{[2]}$. Furthermore, it has been shown that a second group of nociceptors are stimulated only by inflammation and serve to increase pain perception after the original tissue damage. Although unrelated to the original stimulus, decreasing inflammation postoperatively helps to minimize the sensitization of these nociceptors.

Two central components in understanding pain in the postoperative patient are peripheral sensitization and central sensitization.

Peripheral sensitization occurs as the result of the pathways outlined in the previous discussion. Once a patient experiences pain, they can have an increased sensitivity to the same stimulus. This results in hyperalgesia. Allodynia results when a previous stimulus that had ane time not caused pain now does. New synapses are formed with dorsal horn cells that previously received nociceptive input and this redistribution allows mechanoreceptors to activate pain pathways by stimuli that are normally nonnoxious, such as touch ${ }^{[5]}$.

There is augmentation of the initial pain response after the peripheral nociceptors synapse with secondorder neurons in the dorsal horn of the spinal cord. This is the phenomenon of central sensitization. With repeated stimulation by painful stimuli, the second-order neurons become hyper-responsive and exhibit augmented sensitivity. This phenomenon is referred to as "wind up". Chemical mediators such as excitatory amino acids glutamate and aspartate at $N$-methyl- $D$-aspartate (NMDA) result in central hypersensitivity. Repeated peripheral stimuli lead to changes in the dorsal horns of the spinal cord or neuroplasticity, which contributes to increased hypersensitivity to peripheral stimuli ${ }^{[6]}$. It is hypothesized that the irreversible changes which occur in the dorsal horns of the spinal cord in response to repeated peripheral stimuli may be the cause of chronic pain syndromes.

It is generally accepted that postoperative pain is related to many factors, including the amount of soft tissue injury, resulting inflammation, and rib injury (as in the case of a thoracis surgical procedure). There are other individual factors that need to be considered, including, but not limited to, preoperative tolerance to medications, psychological and social factors, and other co-existing morbidities that may or may not contribute to pain (an example of this is fibromyalgia).

\section{PAIN AFTER ROBOTIC THORACIC SURGERY}

Assessment and treatment of the patient undergoing robotic thoracic surgery should utilize the same concepts of peripheral and central sensitization as in any patient experiencing pain after thoracic surgery. 
Furthermore, these concepts should be applied to the specific responses of the individual patient. Many studies have focused on the patient undergoing a conventional thoracotomy. It is generally agreed that thoracotomy is an extremely painful procedure that requires aggressive perioperative and postoperative attention to pain management. Any inattention to pain invariably leads to such deleterious consequences as atelectasis, pneumonia, DVT/PE, and subsequently prolonged hospitalization. To minimize complications, it has been hypothesized that decreasing the size of the incision or "sparing" the muscles of the chest will decrease the resulting pain. This commonly accepted hypothesis has not proven to be true. In fact, a recent study by Ochroch et al. ${ }^{[7]}, 2005$, compared patients undergoing a traditional postero-lateral thoracotomy with those undergoing a muscle-sparing thoracotomy and found no difference in perceived pain up to 48 weeks postoperatively.

There has also been recent work outlining the differences between a traditional thoracotomy and a video-assisted surgery. In 1994, Landreneau compared 165 patients who underwent a postero-lateral thoracotomy and 178 who underwent the Video-assisted Thoracic Surgery (VATS) technique. This study found that less subjective pain was reported by the VATS group in the first year after surgery; however, analgesic requirements were similar ${ }^{[8]}$. In a smaller study also in 1994 , a smaller study reported similar findings comparing the two groups ${ }^{[8]}$. However, in this study, the lower levels of perceived pain by the VATS patients was noted only in the first few days after surgery. These studies are substantiated by more recent ones, such as Li et al ${ }^{[9]}, 2003$, who found that, when compared to the posterolateral thoracotomy, VATS surgery was associated with significantly less shoulder dysfunction and pain medication requirement in the early postoperative period. While some of the reasons for these differences may be attributed in part to the smaller incisions, which presumably result in smaller amount of tissue injury, the entire reasoning is more complex. Referring to the previous discussion about nociception, it is not only the activation of the nociceptors that leads to hyperalgesia, but also the chemical mediators that are released at the same time and contribute to the overall peripheral sensitization. Yim et al. ${ }^{[10]}$ compared thoracotomy to VATS in relation to cytokine response. They found that not only did the VATS group have significantly less analgesic requirement, but also that plasma levels of interleukin 6 and interleukin 8, both pro-inflammatory cytokines, were reduced in the VATS group. Based on this study, it appears that decreased humoral mediators may contribute to decreased sensitization following VATS. In fact, VATS and thoracotomy may be similar as initial stimuli for nociceptors but the advantage of VATS may be due to the lower level of sensitization and lessened response to the initial stimulus.

As robotic thoracic surgery further decreases the invasiveness of thoracic surgery, the principles of pain management with VATS need to be applied and modified for robotic thoracic surgery.

\section{PAIN MANAGEMENT}

\section{Preemptive analgesia}

Successful pain management encompasses choices made in both the perioperative and postoperative periods. Earlier pain control may prevent central sensitization. As explained above, beginning pain management earlier will help to prevent central sensitization. There has been much attention paid recently to the concept of preemptive analgesia. Preemptive analgesia is simply the theory that, by stopping or decreasing the input of stimuli (nociception), one can prevent or decrease central sensitization, and, in turn, achieve a decrease in overall pain. An extension to this concept is the hypothesis that, by administering analgesia prior to nociception, it may be possible to decrease chronic pain syndrome. Electrophysiologic data from animal studies have shown that administering low doses of an opioid such as morphine prior to the introduction of a noxious stimulus can suppress spinal cord hyperexcitability. On the other hand, administering that same opioid after the noxious stimulus does not result in the same degree of $\operatorname{suppression}^{[11]}$. As NMDA is implicated in the "wind up" phenomenon, it is thought that NMDA may play 
a role in preemptive analgesia ${ }^{[11]}$. Consequently, NMDA antagonists such as ketamine or dextromethorphan are possible agents which may result in preemptive analgesia. However, mostly due to study design and variance in the definition of preemptive analgesia, studies comparing pre-incisional and post-incisional pain control have shown inconsistent results.

The timing of preemptive analgesia has been controversial. In its purest sense, preemptive analgesia is that which is applied prior to any stimulation of the nociceptors by any noxious stimulus. However, studies have defined it as pre-surgical $v s$. post-surgical administration ${ }^{[12]}$. Obviously, anesthesia and its attendant procedures represent noxious stimuli to the patient. Newer concepts of preemptive analgesia are based on the realization that the surgical incision alone does not trigger central sensitization, and that other noxious stimuli such as the inflammatory mediators, ectopic neural activity, and preoperative noxious stimuli may play a significant role in the overall pain experience ${ }^{[12]}$. Further studies are required to clarify the appropriate time for preemptive analgesic intervention, which is designed to prevent central sensitization. It is currently unknown what severity or duration of pain is required for sensitization to occur, thus the timing of analgesia is also unknown. Prevention of central sensitization remains the key to a successful strategy for the control of acute and chronic pain.

\section{Options for postoperative pain management}

There are several options for pain management in the postoperative robotic thoracic surgical patient. However, the focus of any regimen should be timing and accurate measurement of pain. Early initiation of therapy is paramount to a successful strategy. In addition, since the goal of robotic, or minimally invasive, surgery is early discharge and a quicker recovery, pain management should be compatible with shorter hospitalization and treatment in the outpatient setting.

Under-treatment of pain remains a problem in both hospital and outpatient settings. A multi-center survey showed that, although patients' satisfaction with pain management had improved from $14 \%$ to $19 \%$, as many as eight out of ten patients reported inadequate pain management ${ }^{[13]}$. This study showed that mobility improved with better pain control ${ }^{[13]}$.

Assessment of pain needs to be accurate and consistent. Although there are many proven approaches, there remains a shortage of knowledge and a lack in consistency and follow-through. Use of a pain scale has been shown to provide a clear method for evaluating and tracking postoperative pain. The visual analog scale has been shown to be an effective tool for measuring surgical pain. Furthermore, it has been shown to be an excellent tool for comparing pain levels between groups at a point in time or to track a single patient's pain and response to interventions ${ }^{[14,15]}$. The intensity of pain should be recorded and reviewed at regular intervals as well as after each intervention, and the same measurement scale for pain should be used across all disciplines, from anesthesia to the bedside nurse.

\section{Systemic pain control}

\section{Opioid administration}

Until recently, opioids have been the mainstay of analgesia in the postoperative robotic surgery patient. They have proven value in managing severe pain. Opioid administration begins intravenously in the perioperative period. It usually continues via intravenous methods until the patient is awake and able to take a diet without difficulty. This can take up to a day depending on the patient's reaction to anesthesia, timing of the surgery, and individual pain perception. Patient controlled analgesia (PCA) is an accepted route of intravenous (IV) opioid administration. It has a high acceptance level among patients and allows for quick and easy administration. However, this technique is not always necessary in the patient undergoing minimally invasive surgery and should be considered on an individual basis. Intravenous opioids should be converted to the oral route as soon as possible. While intravenous opioids have rapid 
onset, they also have shortened duration of action and prolonged use can lead to a "roller coaster" effect of pain followed by relief of pain. Opioids are associated with significant side effects: nausea, vomiting, respiratory compromise, and ileus. Consequently, they are used in a manner which can result in ineffective pain control.

Oral administration is reserved for when the patient can take a diet without difficulty. Transitioning smoothly to an oral regimen is key. A shortcoming of the oral route is the delay in the onset and peak of drug activity. The addition of a long acting opioid will aid in preventing this "peak and valley" phenomenon.

The use of opioids may extend for several days to several weeks and is highly patient dependent. Many practitioners are hesitant to prescribe opioids long term for several reasons. The treatment of pain with opioids and the prevention of the side effects is preferable to the consequences associated with poor pain control, usually stated as side effects of nausea and constipation as well as fear of addiction. All prescribers of opioids have an ethical duty to provide appropriate pain relief to their patients, while taking into account the many societal and political issues that have emerged as the result of opioid over prescription. Obviously, opioids need to represent an adjunct to a more effective pain management strategy.

\section{Nonsteroidal anti-inflammatory agents}

Inflammation is a natural and often protective response to tissue injury caused by surgery. It usually subsides when healing is complete. Inflammation is triggered by the release of chemical mediators, which progress with a cascade effect. Prostaglandins are key mediators in the process of nociception. Prostaglandins are synthesized in the spinal cord and are produced from arachidonic acid via the cyclooxygenase pathway. There are two defined and a third as yet undefined cyclooxygenase enzymes ${ }^{[2]}$. COX-1 is in most cells as well as the peripheral and central nervous systems and is produced a number of pathways. COX-2 is generated to a more limited extent, mostly in the central nervous system. Inhibiting the COX enzyme and thereby decreasing peripheral and central prostaglandin production has been shown: (1) to decrease the inflammation associated with tissue injury; and (2) to decrease peripheral and central sensitization. Zhu and Eisenach ${ }^{[16]}$ demonstrated that there are differences in spinal COX isoenzymes involved in different pain states, with a dominant role for spinal COX-2 with peripheral inflammation and a more exclusive role for COX-1 after incisional surgery. This may have implications for control of hypersensitivity after nerve injury but needs to be shown in humans.

Postoperative use of NSAIDs has been shown to decrease opioid use while still providing adequate analgesia. Furthermore, NSAIDs have little effect on homodynamic parameters, with negligible changes in blood pressure and stroke volume. In addition, in comparison to opioids such as morphine, which has been shown to decrease minute ventilation and increase pulmonary vasoconstriction, NSAIDs have very little effect on pulmonary circulation ${ }^{[2]}$.

The use of NSAIDs may impact renal function. This is especially relevant in thoracic surgical patients who are usually elderly and are subjected to postoperative fluid restriction. However, several studies have not supported this hypothesis. In patients with normal preoperative renal function undergoing thoracic surgery, the use of NSAID was associated with minimal reduction in creatinine clearance and no change in urine output ${ }^{[2]}$. Another concern is the potential for gastrointestinal bleeding associated with NSAID use. While gastrointestinal erosion can be seen with long-term or chronic NSAID use, its incidence has not been proven with short-term perioperative use. However, several studies have shown that, for long-term use, COX-2 inhibitors may be superior to non-selective NSAID ${ }^{[2]}$.

Ketorolac (Toradol), $15 \mathrm{mg}$ every $6 \mathrm{~h}$ (can be intravenously or intramuscularly), is typically the NSAID used in the perioperative period. The intravenous route is a preferred route in the immediate postoperative 


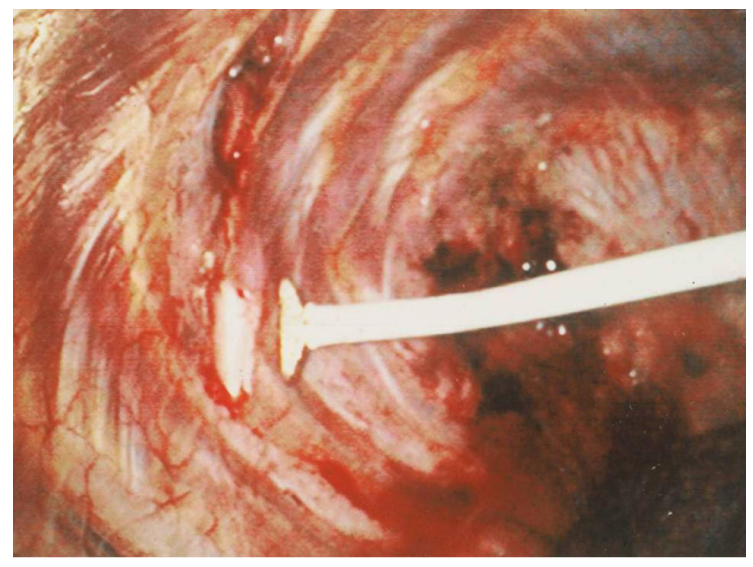

Figure 1. View of the right pleural space. Cryoanalgesia probe being used to freeze the intercostals nerve

period. In addition, ibuprofen, 400-600 mg every 6-8 h, and indomethacin, $25 \mathrm{mg}$ every $8 \mathrm{~h}$, are used. COX-2 inhibitors are less available due to recent studies showing a potential increased rate of cardiovascular thromboembolic events.

\section{Local pain control}

Several strategies have been used for local pain control in thoracic surgical patients.

\section{Epidural analgesia}

Epidural analgesia is a generally accepted form of analgesia in patients undergoing a thoracotomy. The catheter is normally left in place for three or four days. Epidural catheters require constant attention. A percentage of the catheters malfunction and require removal versus replacement. Patients are generally not allowed to bathe or shower until the catheter is removed. Complications include neurologic injury and bleeding around the spinal cord. Hypotension and urinary retention are common side effects ${ }^{[17]}$. Although epidural catheters can provide excellent pain relief, they are not commonly used with VATS because of the time required for insertion, frequent side effects, and the relatively short period of effective use ${ }^{[18,19]}$. Earlier ambulation and shorter hospital stay with VATS and robotic thoracic surgery preclude the use of epidural catheter.

\section{Cryoanalgesia}

In 1999, Detterbeck et al. ${ }^{[19]}$ showed a decrease in perceived pain in patients undergoing VATS surgery with cryotherapy of the intercostal nerves when compared to those undergoing VATS and pain management by epidural catheters and analgesics. In a subsequent study, cryoanalgesia of the intercostal nerves was shown to be effective in preventing post thoracotomy pain syndrome in patients who had undergone VATS ${ }^{[20]}$ [Figures 1 and 2]. However, several studies have shown that cryoanalgesia is associated with long-term complications. Most notably, cryoanalgesia has been associated with long-term neuralgia in the distribution of the treated nerves in up to $12 \%$ of patients ${ }^{[20,21]}$. Although cryoanalgesia was associated with excellent short- and long-term pain control following VATS, it was associated with irreversible hyperesthesia in $8 \%$ of patients. It has been hypothesized that this was due to the inability to control the degree and depth of the cold injury to the nerve, which resulted in irreversible damage and neuralgia ${ }^{[2,23]}$. As a result of this experience, intercostal cryoanalgesia is no longer used in thoracic surgical patients.

\section{Paravertebral blocks}

Intraoperative paraverterbral (subpleural and intercostal) administration of long-acting local anesthetic agents have been used. This technique uses individual intercostal blocks or placement of an indwelling 


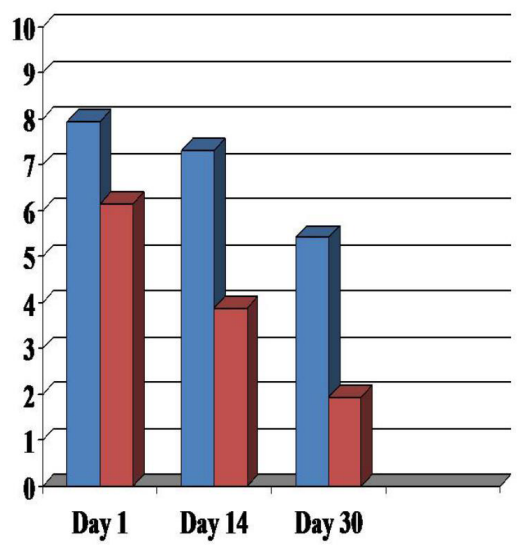

\section{$\square$ Non Cryo Group $\square$ Cryo Group}

Figure 2. Comparison of cryoanalgesia with conventional pain control techniques utilizing Likert Pain Scores. Although the level of the pain is significant, cryoanalgesia is more effective than conventional pain control techniques in controlling pain on Days 1, 14, and 30

catheter. The blocks generally last 18-24 h and are very effective and considered by some equivalent to an epidural in the first $24 \mathrm{~h}^{[24]}$. A major shortcoming of this technique is the variability of catheter or block placement by different practitioners, the extra time required in the operating room, and the inconsistent results from errors in catheter placement.

\section{Liposomal bupivacaine}

Standard bupivacaine maintains local anesthetic effects for approximately $18 \mathrm{~h}$. Liposomal bupivacaine (Exparel) has prolonged local anesthetic effect for up to $72 \mathrm{~h}$. Liposomal bupivacaine is administered using either a transcutaneous or a intrathoracic technique. Liposomal bupivacaine is approved for local administration in surgical incisions; however, many thoracic surgeons are using this medication for subpleural paravertebral blocks in an off-label application. Using this technique, pain relief has been shown to be better than shorter-acting agents and similar to thoracic epidural ${ }^{[24,25}$. In addition, studies have shown decreased postoperative narcotic administration, shorter hospital stays, and better pain scores versus thoracic epidural analgesia ${ }^{[26-29]}$.

\section{Subpleural infusion of local anesthetic}

Presently, most robotic surgeons begin the procedure with infiltration of the intercostal nerve with local anesthetic prior to the conduct of the operation. Other surgeons use local infiltration of the intercostals at the end of the procedure as their preferred method of local pain control. One shortcoming of this technique is that the local pain control is short lived and the effect of the local anesthetic quickly wears off.

On the other hand, multiple studies have shown that the continuous infusion of local anesthetic through a catheter placed in an extra pleural tunnel overlying the intercostal nerves to be safe and efficacious ${ }^{[30]}$. The advantage of this technique is prolonged local pain control. Some investigators have reported placing the catheter in an extrapleural pocket, while others have placed them close to the heads of the ribs in the paravertebral space ${ }^{[30]}$. Various types of catheters have been used. Randomized studies have demonstrated better pain relief, better pulmonary function, lower pulmonary complications, and lower use of narcotics with the use of extrapleural infusion catheters ${ }^{[31-34]}$. Studies are bearing out the efficacy of subpleural infusion of local anesthetic in the acute setting. Taylor et al ${ }^{[33]}$ specifically studied the use of this technique in minimally invasive surgery and found it to be an effective form of analgesia and to decrease narcotic requirements postoperatively. In addition, Concha et al ${ }^{[34]}$ studied the use of intercostal nerve blockade combined with IV PCA compared to epidural analgesia and found little statistical significance between the two groups. Detterbeck reviewed studies on extrapleural catheter use in patients undergoing 


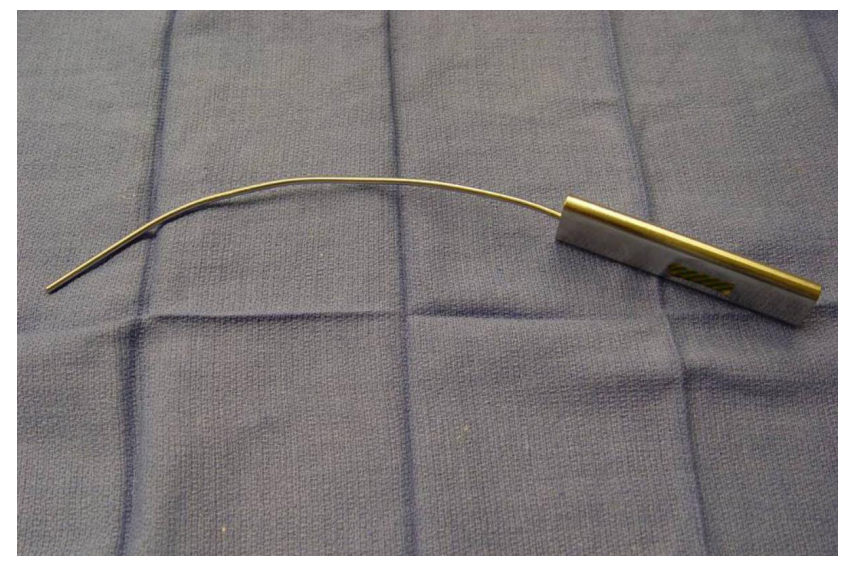

Figure 3. Tunneler for subpleural placement of local anesthetic catheters

thoracotomy and found that the use of extrapleural catheter for analgesia was superior to systemic narcotics ${ }^{[30]}$. In addition, the use of extrapleural catheters resulted in lower narcotic consumption and decreased pulmonary complications. DiMaio et al.$^{[35]}$ compared the use of a local infusion of an anesthetic to an epidural catheter and found not only improved pain and decreased narcotic usage, but also improved pulmonary function, as demonstrated by an increase in lung volumes. Choice of local anesthetic is surgeon dependent. Moreover, the above-mentioned review did not find a difference in pain relief or postoperative complications when comparing bupivacaine, lidocaine, and lignocaine ${ }^{[30]}$. Complications related to the catheter and the local anesthetic agents are low. Reported complications have been less than $0.6 \%$ and have included: transient hypotension, transient Horner's syndrome from placement of catheters above the third intercostal space, transient ipsilateral femoral nerve dysfunction from placement of catheters lower than the eighth intercostal space and infusion of the local anesthetic into the retroperitoneum, bupivacaine toxicity in the form of confusion, transient elevation of liver enzymes, and rib osteomyelitis ${ }^{[30]}$.

Technique for the placement of subpleural catheters after robotic surgery. https://youtu.be/2JaF3j4re40; https://youtu.be/b49GXgEmyZM

The video of this technique can be accessed using the above links. Although several techniques have been described, we have devised a rapid and reproducible technique for the extrapleural placement of the catheters. With this technique, two soaker catheters are inserted through a subpleural tunnel that extends from the second to the eighth intercostal spaces and encompasses the area of the trocars.

Following the completion of the robotic procedure and undocking of the robot, the camera trocar is removed. An endoscopic camera (Olympus Endoeye o Degree) is introduced through the anterior port and used to visualize the paravertebral pleura. In this technique, a specially designed tunneling device is introduced through the camera port and used to begin the formation of a subpleural tunnel. After the formation of the tunnel, the metal tunneling device is withdrawn and a peelable sheath is positioned over the tunneler and replaced in the pleural tunnel. The metal tunneler is withdrawn and the sheath is left in place inside the pleural tunnel. Two five-inch on-Q soaker catheters are introduced through separate puncture sites placed anteriorly in the same intercostal space as the inferior incision [Figure 3]. The on- $\mathrm{Q}$ soaker catheters are passed into the long subpleural sheath, and then the sheath is withdrawn and peeled away, leaving the soaker catheters in the subpleural tunnel. The catheters are positioned in an overlapping staggered manner to provide infusion of the local anesthetic for the entirety of the pleural tunnel extending from the second to the eighth intercostal spaces. We use the on-Q Pain Buster soaker catheters (I-Flow Corporation, Lake Forest, CA), which are small flexible catheters with multiple side holes that can deliver 


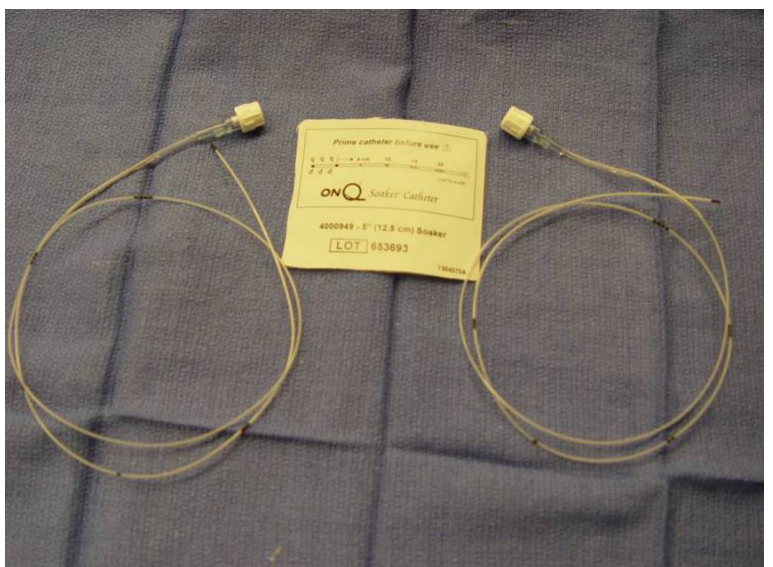

Figure 4. Five-inch on- $Q$ soaker catheters
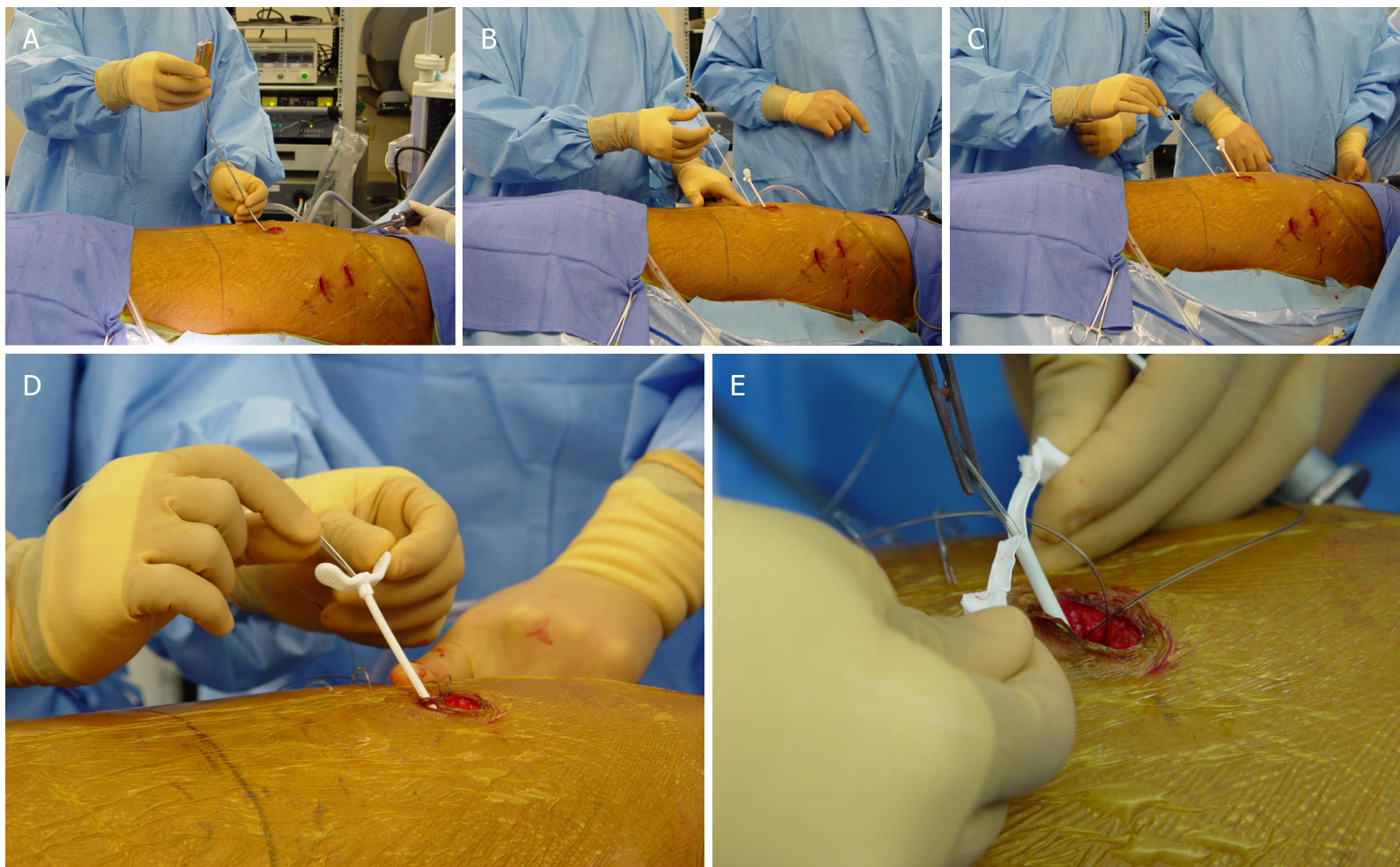

Figure 5. (A-E) Steps for the placement of subpleural catheters for local infiltration of local anesthetic for 10 days in the ambulatory setting

the infusion over multiple areas [Figures 4-6]. With the on-Q system, flow rate and duration are dependent upon the model used, and can range from 0.5 to $10 \mathrm{~mL} / \mathrm{h}$ with a reservoir volume of $65-400 \mathrm{~mL}$. For robotic thoracic surgery applications, we use two catheters, an infusion of approximately $4 \mathrm{~mL} / \mathrm{h}$ ( $2 \mathrm{~mL}$ per catheter) with a $400 \mathrm{~mL}$ reservoir and 0.125 bupivacaine. This system is used after the patient is discharged from the hospital, giving the patient 10 days of local pain control. In our institution, intercostal nerve blockade by infusion of a local anesthetic via a subpleural catheter has been shown to be an effective alternative to epidural catheters and cryoanalgesia. This technique provides excellent prolonged pain control after robotic thoracic surgery while decreasing the need for narcotics [Table 1] ${ }^{[36]}$. 


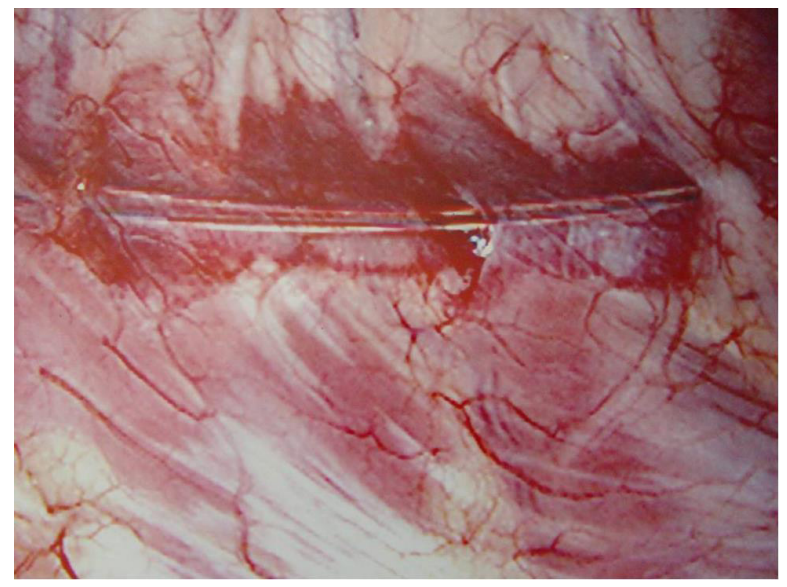

Figure 6. On-Q catheters in place in the narrow subpleural tunnel

Table 1. Likert Pain Scores Median (Range), Subpleural local anesthetic $(n=243)$ vs. Epidural catheter supplemented by narcotics $(n=238)$

\begin{tabular}{llll}
\hline & Day 1 & Day 14 & Day 30 \\
\hline Epidural & $8(4-9)$ & $7(5-9-8)$ & $5(1-5)$ \\
Subpleural Catheter & $2(1-3)$ & $1(1-3)$ & $1(0-1)$ \\
& $P<0.001$ & $P<0.001$ & $P<0.001$ \\
\hline
\end{tabular}

\section{CONCLUSION}

A combined, multimodal approach appears to be the most effective one in dealing with analgesia in the thoracic surgical patient. Any treatment modality needs to attempt to decrease the overall pain experience by preventing sensitization at any time throughout the perioperative course. Thus, multimodal therapy means focusing on addressing pain at the various sites. A multimodal program may embrace two or more therapies. For example, use of neural blockade, whether by epidural or other nerve blocks, is combined with systemic opioid administration (first intravenously, and then via oral route). In addition, NSAID use such as ketorolac in either the preoperative or postoperative phase can also add to the multimodal effect. Choice of a modality is dependent upon many factors, including surgeon preference, anesthesia preference, institutional features or limitations, and personal success or failures with certain treatments. Regardless, a comprehensive pain management regimen is essential for any robotic thoracic surgical program.

\section{DECLARATIONS}

\section{Authors' contributions}

The author contributed solely to the article.

\section{Availability of data and materials}

Not applicable.

\section{Financial support and sponsorship}

None.

\section{Conflicts of interest}

The author declared that there are no conflicts of interest.

\section{Ethical approval and consent to participate}

Not applicable. 


\section{Consent for publication}

Not applicable.

\section{Copyright}

(c) The Author(s) 2020.

\section{REFERENCES}

1. International Association for the Study of Pain. Classification of chronic pain: descriptions of chronic pain syndromes and definitions of pain terms. Pain 1986;3:S1-226.

2. Ochroch EA, Gottschalk A. Impact of acute pain and its management for thoracic surgical patients. Thorac Surg Clini 2005;15:105-21

3. Raja SN, Dougherty PM. Pain and the neurophysiology of somatosensory processing. In: Benton HT, Raja SN, editors. Essentials of pain medicine and regional anesthesia. New York: Churchill Livingstone; 2001. pp. 2-6.

4. Johnson BW. Pain mechanisms: anatomy, physiology, and neurochemistry. In: Practical management of pain. 3rd ed. St. Louis, Mosby. 2000. pp. 117-43.

5. Marcus DA. Treatment of nonmalignant chronic pain. Am Fam Physician 2000;61:1331-8.

6. Erdek MA, Staats PS. Chronic pain and thoracic surgery. Thorac Surg Clin 2005;15:123-30.

7. Ochroch EA, Gottschalk A, Augoustides JG, Aukburg SJ, Kaiser LR, et al. Pain and physical function are similar following axillary, muscle-sparing vs posterolateral thoracotomy. Chest 2005;128:2664-70.

8. d'Amours RH, Riegler FX, Little AG. Pathogenesis and management of persistent postthoracotomy pain. Chest Surg Clin N Am 1998;8:703-22.

9. Li WW, Lee TW, Yim AP. Shoulder function after thoracic surgery. Thorac Surg Clin 2004;14:331-43.

10. Yim APC, Wan S, Lee TW, Arifi AA. VATS lobectomy reduces cytokine responses compared with conventional surgery. Ann Thorac Surg 2000;70:243-7.

11. Soto RG, Fu ES. Acute pain management for patients undergoing thoracotomy. Ann Thorac Surg 2003;75:1349-57.

12. Katz J, Cohen L, Schmid R, Chan VWS, Wowk A. Postoperative morphine use and hyperalgesia are reduced by preoperative but not intraoperative epidural analgesia. Anesthesiology 2003;98:1449-60.

13. Miller EH, Belgrade MJ, Cook M, Portu JB, Shepherd M, et al. Institutionwide pain management improvement through the use of evidence-based content, strategies, resources, and outcomes. Qual Manag Health Care 1999;7:28-40.

14. McCarthy M, Chang C, Pickard AS, Giobbie-Hurder A, Price DD, et al. Visual analog scales for assessing surgical pain. J Am Coll Surg 2005;201:245-52.

15. Gordon DB. Critical pathways: a road to institutionalizing pain management. J Pain Symptom Manage 1996;11:252-9.

16. Zhu X, Eisenach JC. Cyclooxygenase-1 in the spinal cord is altered after peripheral nerve injury. Anesthesiology 2003;99:1175-9.

17. Gerner P. Post-thoracotomy pain management problems. Anestheisiol Clin 2008;26:355-7.

18. Cense HA, Lagarde SM, de Jong K. Association of no epidural analgesia with postoperative morbidity and mortality after thoracic esophageal cancer resection. J Am Coll Surg 2006;202:395-400.

19. Detterbeck FC. Subpleural catheter placement for pain relief after thoracoscopic resection. Ann Thorac Surg 2006;81:1522-3.

20. Tempesta B, Gharagozloo F, Birenberg A, Kim Y, Trachiotis G, et al. Cryonalagesia for relief of pain after video-assisted thoracic surgery. Chest 1999;116.

21. Salameh J, Tempesta B, Gharagozloo F, Birenberg A, Kim Y, et al. Cryoanalgesia for relief of pain after video-assisted thoracic surgery. Chest 2001.

22. Johannesen N, Madsen G, Ahlburg P. Neurological sequelae after cryoanalgesia for thoracotomy pain relief. Ann Chir Gynaecol 1990;79:108-9.

23. Khanbhai M, Yap KH, Mohamed S, Dunning J. Is cryoanalgesia effective for post-thoracotomy pain? Interact Cardiovasc Thorac Surg 2014;18:202-9.

24. Jackson SM, Whitlark JD. Pain control with liposomal bupivacaine after thoracoscopies/thoracotomies. Ann Thoracic Surg 2015;100:2414-5.

25. Rice DC, Cata JP, Mena GE, Rodriguez-Restrepo A, Correa AM, et al. Posterior intercostal nerve block with liposomal bupivacaine: an alternative to thoracic epidural analgesia. Ann Thorac Surg 2015;99:1953-60.

26. Dominguez D, Bach CT, Lee TM, Velotta JB. Impact of liposomal bupivacaine on length of hospitalization in thoracic surgery patients. J Am Coll of Surg 2017;225:S30-1.

27. Parascandola SA, Ibañez J, Keir G, Anderson J, Plankey M, et al. Liposomal bupivacaine versus bupivacaine/epinephrine after videoassisted thoracoscopic wedge resection. Interact Cardiovasc Thorac Surg 2017;24:925-30.

28. Dasta J, Ramamoorthy S, Patou G, Sinatra R. Bupivacaine liposome injectable suspension compared with bupivacaine $\mathrm{HCl}$ for the reduction of opioid burden in the postsurgical setting. Curr Med Res Opin 2012;28:1609-15.

29. Sparks A, Stewart J. Review of pain management in thoracic surgery patients 2018. J Anesth Clin Res 2018;9:817.

30. Detterbeck FC. Efficacy of methods of intercostal nerve blockade for pain relief after thoracotomy. Ann Thorac Surg 2005:80:1550-9.

31. Wheatley GH, Rosenbaum DH, Paul MC, Dine AP, Wait MA, et al. Improved pain management outcomes with continuous infusion of a local anesthetic after thoracotomy. J Thorac Cardiovasc Surg 2005;130:464-8. 
32. Duarte AM, Pospisilova E, Reilly E, Mujenda F, Hamaya Y, et al. Reduction of postincisional allodynia by subcutaneous bupivicaine. Anesthesiology 2005;103:113-25.

33. Taylor R, Massey S, Stuart-Smith K. Postoperative analgesia in video-assisted thoracoscopy: the role of intercostal blockade. J Cardiothorac Vasc Anesth 2004;18:317-21.

34. Concha M, Dagnino J, Cariaga M, Aquilera J, Aparicio R, et al. Analgesia after thoracotomy: epidural fentanyl/bupivacaine compared with intercostal nerve block plus intravenous morphine. J Cadriothorac Vasc Anesth 2004;18:322-6.

35. Wheatley GH, Rosenbaum D, Paul MC, Dine AP, Wait MA, et al. Improved pain management outcomes with continuous infusion of local anesthetic after thoracotomy. JTCVS 2005;130:464-8.

36. Tempesta BJ, Gharagozloo F, Margolis M, Strother E. Prolonged subpleural infusion of local anesthetic for pain relief after thoracic surgery. Chest 2007;13:661A. 